\title{
Modèles d'évolution de lits alluvionnaires soumis à charriage : Tri granulométrique et pavage
}

\author{
Jean-Luc Rahuel Sogreah, B.P. 172 X, 38042 Grenoble Cedex \\ Jean-Pierre Chollet Institut de mécanique de Grenoble *, B.P. 53 X, 38041 Grenoble Cedex
}

1. Simulation d'évolutions instationnaires de fonds de rivières

Les problèmes d'évolution à grande échelle de temps et d'espace (plusieurs dizaines voire centaines de kilomètres, pour des périodes qui peuvent s'étendre sur plusieurs années) ne peuvent, en général, pas être traités par l'utilisation du modèle physique. Le modèle mathématique s'impose alors comme l'outil de simulation.

L'étude présentée ici s'intègre aux travaux de mise au point du code CARICHAR, dont certains aspects, plus particulièrement la couche mélangée et le pavage, sont évoqués dans [8]; pour une description exhaustive du modèle et de ses applications, on se reportera à [7].

Le système d'équations sur lequel est construit le modèle mathématique est constitué :

(i) d'équations aux dérivées partielles qui expriment, de manière que l'on peut considérer comme rigoureuse, des propriétés de conservation et de bilan à l'échelle d'un tronçon élémentaire de rivière,

\footnotetext{
* Institut national polytechnique et Université Joseph Fourier de Grenoble, unité associée au CNRS UA 006.
}

(ii) de relations complémentaires, d'origine largement empirique.

Pour pouvoir traiter des problèmes de tri granulométrique et de pavage, le matériau solide n'est pas considéré comme ayant des caractéristiques uniformes : il est divisé ("discrétisé ») en $K$ tranches granulométriques $k$ auxquelles seront attachées les variables selon $x$ et $t$ :

(a) $\beta_{k}$, fraction du volume de sédiments transportés dont le diamètre moyen est $d_{k}$;

(b) $\beta_{k}^{(b)}$ fraction du volume de sédiments en place dans la couche mélangée du lit (de section $A_{m}$ ), dont le diamètre moyen est $d_{k}$.

\subsection{Equations de conservation et de bilan}

Les relations sont écrites dans le cadre d'une hypothèse d'écoulement «filaire ", c'est-à-dire unidimensionnel, selon la seule direction d'espace $x$.

La continuité de l'écoulement implique :

$$
\frac{\partial A}{\partial t}+p \frac{\partial A_{s}}{\partial t}+\frac{\partial Q}{\partial x}=0
$$

où $A$ est la section de l'écoulement, $A_{s}$ la section soumise à dépôt ou érosion, $Q$ le débit liquide.

\section{Alluvial river modelling : bed load, grain sorting and armouring}

An unidimensional model of alluvial river is developed to take account of temporal and spatial evolutions of bed (and bed load) grain compositions. The set of equations is solved in a fully coupled manner; numerical coefficients of the implicit scheme allow to optimize the time step value. A bed load "loading law" is included in the model. Some examples derived from the general features of the " middle third" of the Rhône river make clear how the flow unsteadiness affects the bed evolutions, through grain sorting and bed armouring. 
Le bilan de quantité de mouvement conduit à :

$$
\frac{\partial Q}{\partial t}+\frac{\partial}{\partial x}\left(\frac{Q^{2}}{A}\right)+g A \frac{\partial y}{\partial x}+g A S_{f}=0
$$

où $y$ est la cote de la surface libre et $S_{f}$ la pente de la ligne d'énergie.

La conservation de la quantité de matière solide totale transportée s'écrit :

$$
(1-p) \frac{\partial A_{s}}{\partial t}+\frac{\partial A_{c h}}{\partial t}+\sum_{k=1}^{K} \frac{\partial G_{k}}{\partial s}=0
$$

où $p$ est la porosité de la section $A_{s}$, et $A_{c h}$ la section à travers laquelle s'effectue le charriage; $G_{k}$ est le débit solide de la fraction granulométrique $k$.

Les échanges entre phase solide transportée et fond s'opèrent à l'intérieur d'une couche «mélangée " et la conservation détaillée de chaque fraction granulométrique $k$ s'exprime par :

$$
\begin{aligned}
\frac{\partial G_{k}}{\partial x}+(1-p) A_{m} \frac{\partial \beta_{k}}{\partial t}+(1-p) X_{k} \frac{\partial A_{m}}{\partial t}+ & \\
& +(1-p) Y_{k} \frac{\partial A_{s}}{\partial t}=0
\end{aligned}
$$

avec

$$
\begin{aligned}
X_{k} & =\beta_{k}-\varepsilon_{1}\left(\varepsilon_{2} \beta_{k}+\left(1-\varepsilon_{2}\right) \beta_{k}^{(b)}\right) \\
Y_{k} & =\varepsilon_{1}\left(\varepsilon_{2} \beta_{k}+\left(1-\varepsilon_{2}\right) \beta_{k}^{(b)}\right)
\end{aligned}
$$

$\varepsilon_{1}=0$ en cas de dépôt et $\varepsilon_{1}=1$ en cas d'érosion ; $\varepsilon_{2}=0$ si la couche mélangée absorbe des particules de la sous-couche du lit en place, $\varepsilon_{2}=1$ dans le cas contraire.

Contrairement à des modèles antérieurs [3], [4], les échanges avec le fond sont exprimés sous forme d'une équation aux dérivées partielles, ce qui permet une résolution totalement couplée du système des équations, ainsi que la prise en compte de ces échanges dans l'analyse des directions caractéristiques du système et des vitesses typiques de propagation.

\subsection{Relations complémentaires, d'origine semi- empirique}

Pour fermer le système d'équations, il faut ajouter des relations :

- la dissipation d'énergie pour laquelle la relation la plus générale serait de la forme :

$$
R_{s}\left(S_{f}, Q, A, \beta_{k}, \beta_{k}^{(b)}, G_{k}, \ldots\right)=0
$$

dans les exemples traités ici, on a retenu la formulation simple de Strickler avec une rugosité $K_{s t}$ constante.

- L'intensité du transport solide pour chacune des fractions $k$ représentative de la granulométrie

$$
R_{G}^{(k)}\left(G_{k}, S_{f}, Q, A, \beta_{k}, \beta_{k}^{(b)}, \ldots\right)=0
$$

pour laquelle on a retenu une formulation de Meyer Peter étendue à l'écriture du transport $G_{k}$ pour chacune des fractions.

On notera que des formulations aussi simples ne peuvent décrire qu'imparfaitement les phénomènes com- plexes qui prennent leur origine dans des interactions, fortement non linéaires, entre écoulement, phase solide transportée et fond en place ; la turbulence joue un rôle majeur dans tous ces phénomènes.

\subsection{Loi de chargement}

Les formules de transport solide sont supposées fournir une valeur «potentielle" du débit $G^{*}$, valeur qui correspondrait au transport observé dans un écoulement uniforme sur un lit en équilibre. Le débit solide réel $G$ peut s'écarter notablement de la valeur potentielle $G^{*}$, par exemple immédiatement à l'aval d'un barrage assurant une rétention totale du matériau solide. La loi dite de « chargement " est alors introduite pour exprimer l'écart entre ces valeurs effective et potentielle.

Cette loi de chargement a été introduite dans le code sous la forme :

$$
\frac{\partial G}{\partial x}=K_{1}\left(G^{*}-G\right)+\varepsilon_{c} \frac{G}{G^{*}} \frac{\partial G^{*}}{\partial x}
$$

de façon à utiliser la loi de DAUBERT et LEBRETON [2] pour $\varepsilon_{c}=0$ et la loi de Bell et SuTHERLAND [1] pour $\varepsilon_{c}=1$.

La constante $K_{1}$ n'est autre que l'inverse d'une distance caractéristique de la "relaxation" introduite par cette loi. A l'utilisation de ces lois dans le code numérique, il apparaît, que pour une discrétisation spatiale et temporelle assurant stabilité et convergence, la sensibilité à la valeur de cette constante est faible pour la loi de SUTHERLAND et relativement importante pour la loi de DAUBERT et LEBRETON.

\section{Etude du système d'équations et méthode numérique}

\subsection{Recherche et analyse des directions caractéristiques}

La prise en compte de la loi de chargement se traduit par une modification de la nature du système d'équations à résoudre. Si on considère le cas « simple » d'une granulométrie décomposée en deux fractions $(K=2)$, on trouve 7 directions caractéristiques, schématisées sur la figure 1 , dont les pentes peuvent être interprétées comme des célérités de propagation :

- $\lambda_{1}$ et $\lambda_{2}$, célérités des ondes liquides que l'on connaît dans les problèmes classiques d'écoulements sur fonds fixes,

- $\lambda_{3}$ et $\lambda_{4}$, célérités des ondes « solides »; $\lambda_{4}=0$ si une des fractions granulométriques est immobile, par ailleurs, ce sont $K$ caractéristiques qu'il faudrait considérer pour une décomposition en $K$ fractions,

- $\lambda_{\infty 1}$ et $\lambda_{\infty 2}$ célérités infinies qui traduisent le caractère dégénéré des équations de chargement dans lesquelles ne figure aucune dérivée temporelle

- $\lambda_{0}=0$ dû à la conservation du matériau solide $\left(\beta_{1}+\beta_{2}=0\right)$.

Les expressions de certaines de ces célérités sont directement dépendantes des formulations du transport solide et du choix de la loi de chargement. 
L'observation de ces directions caractéristiques montre qu'il faut spécifier, en régime subcritique: (i) deux conditions aux limites, amont et aval, pour l'écoulement ; (ii) des conditions à l'amont pour le transport solide, les caractéristiques relatives aux "ondes solides " étant en général de pente positive ; (iii) une condition initiale qui influencera directement toutes les solutions du fait de la caractéristique verticale; (iv) des conditions associées aux directions horizontales et que nous choisirons d'imposer à l'amont.

\subsection{Modélisation aux différences finies}

Un modèle élaboré précédemment [3], [4], possédait déjà bien des éléments du modèle présenté ici, particulièrement en ce qui concerne les lois de transport solide et l'utilisation d'une couche mélangée ; cependant, il présentait les inconvénients de ne pas tenir compte des "ondes liquides" ni de traiter de manière couplée l'équation de la couche mélangée. Ces limitations conduisaient, de fait, à avoir des problèmes mathématiquement mal posés, rendant incertain le choix approprié de conditions aux limites et pénalisant les propriétés de conservation du calcul.

Les équations sont approchées par des différences finies selon un schéma de PrEISSMANN généralisé, avec pondérations spatiale et temporelle, toute variable $f$ pouvant alors s'écrire entre les instants $n \Delta t$ et $(n+1) \Delta t$, et entre les sections d'abscisse $j \Delta x$ et $(j+1) \Delta x:$

$$
\begin{aligned}
f=\psi\left((1-\theta) f_{j+1}^{n}+\theta f_{j+1}^{n+1}\right)+ & \\
& +(1-\psi)\left((1-\theta) f_{j}^{n}+\theta f_{j}^{n+1}\right) .
\end{aligned}
$$

Des études de ce schéma dans le contexte de modélisation alluviale [5], [6], ont été complétées par la prise en compte de la loi de chargement et la représentation de la composition granulométrique. Les valeurs $\psi=0,5$ et $\theta=0,5$ apparaissent comme des limites des domaines de stabilité ; les limites de ces domaines peuvent être précisées en considérant les diverses célérités $\lambda$ calculées au cours de l'étude des directions caractéristiques. On met en évidence [7] l'intérêt pratique d'envisager des pondérations $(\psi, \theta)$ différentes pour les équations de l'écoulement et les équations du transport solide puisque coexistent deux échelles de temps significativement différentes (dont le rapport $\tau_{\mathrm{cc}} / \tau_{\mathrm{sol}}$. est de l'ordre de $G / Q)$.

La conservation du matériau solide, globale et détaillée (c'est-à-dire par fraction $k$ ) a été vérifiée systématiquement: les erreurs ne dépassent pas, en valeur relative, $2 \cdot 10^{-4}$ après 720 jours de simulation.

\section{Illustration sur une évolution instationnaire}

Le comportement du modèle mathématique a été analysé sur un exemple inspiré de la morphologie du Rhône central. La granulométrie a été divisée en $K=3$ fractions. Les qualités spécifiques du modèle mathématique sont particulièrement bien mises en évidence dans un calcul d'évolution instationnaire. Parmi les nombreuses variables dont on peut suivre l'évolution spatio-temporelle (voir [7] pour une description complète), nous avons sélectionné, sur la figure 2, la distribution des débits solides par fraction granulométrique, $G_{k}$, à différents instants. On constate que les sédiments les plus fins sont mobiles pour toutes les valeurs du débit, les sédiments moyens ne sont mis en mouvement que pour la pointe de la crue, les sédiments les plus gros restent immobiles et assurent ainsi le pavage du lit.

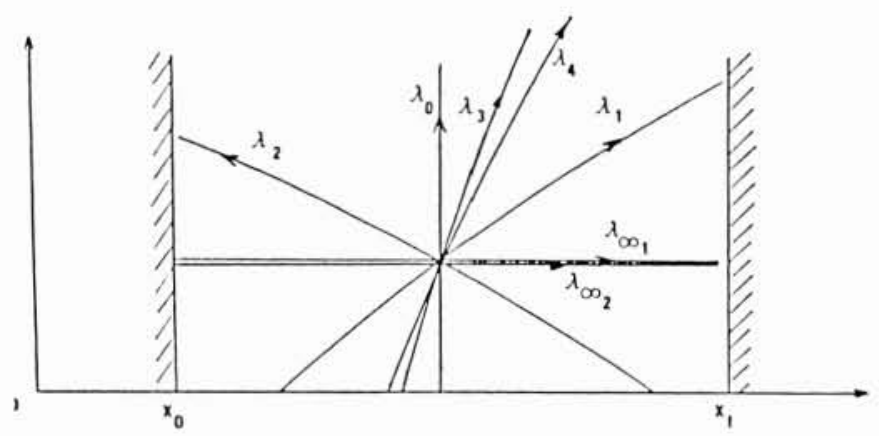

1. Directions caractéristiques dans le cas d'une granulométrie étendue représentée par deux fractions $(K=2)$.

2. Distribution le long du chenal des intensités des transports solides, $G_{k}(x, t)$, pour chacune des 3 fractions granulométriques aux temps 2, 20 et 43 jours, avec la loi de chargement de DAUBERT et LEBRETON, pour une séquence de débits en évolution instationnaire inspirée d'une crue du Rhône (année 1955), avec des barrages en condition limite amont et condition limite aval. (Classe 1: $5-25 \mathrm{~mm}$, classe 2: 25 $70 \mathrm{~mm}$, classe 3 : $70-150 \mathrm{~mm}$.)

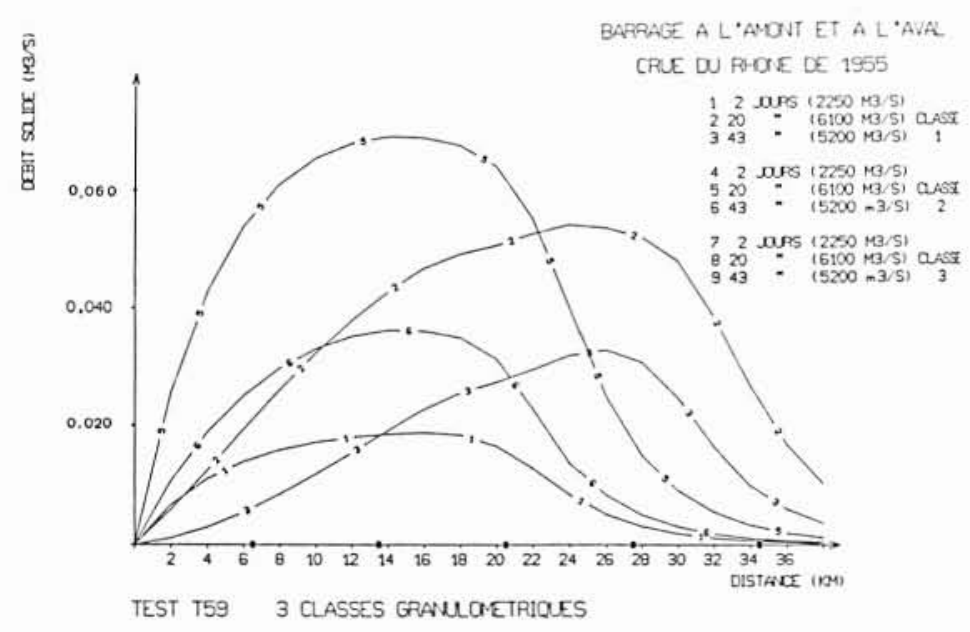




\section{Conclusions}

La modélisation mathématique est un outil adapté aux simulations des évolutions instationnaires des rivières, à grande échelle de temps et d'espace, tout particulièrement lorsque de nouveaux paramètres, tels que les compositions granulométriques, sont pris en compte. L'étude des directions caractéristiques met bien en évidence les modifications profondes que peut subir le modèle lorsque l'on introduit la description de la granulométrie et une loi de chargement. Cette loi de chargement ne paraît pas être vraiment indispensable à la représentation des phénomènes dès l'instant que la discrétisation spatiale est assez fine ; on a par ailleurs noté que cette loi modifie profondément la nature du système des équations à résoudre.
Le caractère couplé et implicite du modèle autorise des pas de temps de valeur assez grande malgré les différences très importantes entre les valeurs des temps caractéristiques des ondes liquides et solides. Par ailleurs cette approche conduit à mathématiquement «bien poser " le problèmé et à spécifier les bonnes conditions aux limites et initiales.

Il faudra valider le code numérique sur des écoulements naturels bien documentés, tout en sachant que, dans les meilleurs des cas, les mesures seront souvent limitées aux relevés des positions de surface libre et de fond. L'amélioration de la modélisation mathématique passera donc aussi par une étude fine, en grande partie expérimentale, de phénomènes de base : mécanique des échanges dans la couche mélangée, lois de dissipation, relations de transport solide relative à une partie de la distribution granulométrique, ...

\section{References}

[1] Bell, R. G., Sutherland, A. J., 1983, Non equilibrium bedload transport by steady flow, Jour. of Hyd. Eng., ASCE, vol. 109, n` 3, 351-367.

[2] Daubert, A., Lebreton, J. C., 1967, Etude expérimentale et sur modèle mathématique de quelques aspects des processus d'érosion des lits alluvionnaires, en régime permanent et non permanent, $12^{\mathrm{c}}$ congrès AIRH.

[3] El SAdig, Mohamed Abdalla Sharfi, 1986, Modélisation mathématique d'évolution des rivières avec prise en compte de la composition granulométrique des matériaux transportés, thèse de l'INPG, Grenoble.

[4] El Sadig, Mohamed Abdalla Sharfi, Chollet, J. P. and BouvARD, M., 1986, Mathematical modelling of alluvial river-bed phenomena, Hydrosoft 86 -hydraulic eng. software, proc. of the 2 nd int. conf., Comput. Mec. pub. Springer Verlag, 103-207.

[5] LYN, D. A., 1987, Unsteady sediment transport modeling, Jour. of Hyd. Eng., ASCE, vol. 113, n* 1, 1-15.

[6] LyN, D. A., Goodwin, P., 1987, Stability of a general Preissmann scheme, Jour. of Hyd. Eng., ASCE, vol. 113, nं $1,16-28$.

[7] Rahuel, J. L., 1988, Modélisation de l'évolution du lit des rivières alluvionnaires à granulométrie étendue, thèse de I'INPG, Grenoble.

[8] Rahuel, J. L. et Belleudy, P., 1988, La simulation numérique des phénomènes de tri granulométrique, présenté à ces Journées de la SHF. 